Revista Arbitrada Interdisciplinaria KOINONIA

Año VI. Vol VI. N 12. Julio - Diciembre. 2021

Hecho el depósito de Ley: FA2016000010 ISSN: 2542-3088

FUNDACIÓN KOINONIA (F.K). Santa Ana de Coro. Venezuela.

María Laura Arteaga-García; María Hercilia Menéndez-Mera

http://dx.doi.org/10.35381/r.k.v6i12.1381

\title{
Redes Sociales: Recurso innovador para emprendedores
}

Social Media: Innovative resource for entrepreneurs

\author{
María Laura Arteaga-García \\ marialauragarcia102@gmail.com \\ Instituto Tecnológico Superior Paulo Emilio Macías, Portoviejo \\ Ecuador \\ https://orcid.org/0000-0001-8071-2347 \\ María Hercilia Menéndez-Mera \\ itspem.mmenendez@gmail.com \\ Instituto Tecnológico Superior Paulo Emilio Macías, Portoviejo \\ Ecuador \\ https://orcid.org/0000-0003-3779-2286
}

Recepción: 01 de mayo 2021

Revisado: 10 de mayo 2021

Aprobación: 15 de junio 2021

Publicación: 01 de julio 2021 


\title{
RESUMEN
}

La investigación tiene por objetivo analizar las redes sociales como recurso innovador para emprendedores de la parroquia Andrés de Vera del cantón Portoviejo, provincia de Manabí-Ecuador. La metodología empleada es de tipo descriptiva con diseño no experimental, en una La población de tipo finita conformada por 140 emprendedores. Facebook, Instagram, figuran como las plataformas de mayor uso por parte de los usuarios o potenciales compradores. Instagram, se constituye en una plataforma que permite no solo promover el negocio, sino, interactuar socialmente con los clientes. El principal reto de los emprendedores, es posicionar su negocio en una sociedad competitiva, donde las marcas consolidadas, tienen la primera opción de compra, por lo tanto, deben diseñar estrategias de venta, pero sobre todo de confianza, credibilidad, para atraer al público.

Descriptores: Comercio electrónico; comercio interno; política comercial. (Palabras tomadas del Tesauro UNESCO).

\begin{abstract}
The objective of the research is to analyze social networks as an innovative resource for entrepreneurs in the Andrés de Vera parish of the Portoviejo canton, province of ManabíEcuador. The methodology used is descriptive with a non-experimental design, in a finitetype population made up of 140 entrepreneurs. Facebook, Instagram, are listed as the platforms most used by users or potential buyers. Instagram is a platform that allows not only to promote the business, but also to interact socially with customers. The main challenge for entrepreneurs is to position their business in a competitive society, where consolidated brands have the first purchase option, therefore, they must design sales strategies, but above all trust, credibility, to attract the public. .
\end{abstract}

Descriptors: Electronic commerce; domestic trade; trade policy. (Words taken from the UNESCO Thesaurus). 


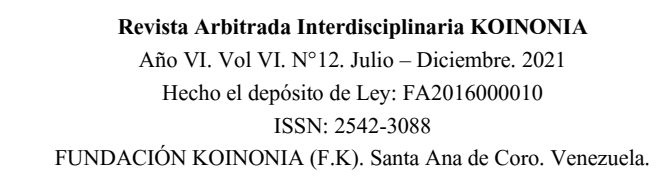

María Laura Arteaga-García; María Hercilia Menéndez-Mera

\section{INTRODUCCIÓN}

Las relaciones sociales de las personas ha sido la esencia innata y natural desde el inicio de la humanidad, pues el hombre fue creado para vivir en sociedad y para ello debe valerse de la comunicación la cual ha evolucionado a pasos agigantados que han permitido grandes cambios en todos los campos permitiendo el desarrollo de los pueblos. Cuando surgió la escritura se convirtió en el primer paso para ampliar la comunicación la cual sería el canal para emprender los intercambios o trueques que fue la primera forma de emprendimiento de negocio, luego aparece otros avances tecnológicos que más allá de una comunicación social, se convirtió en una estrategia para innovar el comercio, lo cual para (Garzón \& Ibarra, 2017), la innovación significa cambiar o alterar algo con un toque novedoso, es decir, que se cambió la forma de emprender un negocio más aún cuando a mediados de los años 60 aparece la Red Internacional, la misma que en la actualidad se conoce como Internet la misma que poco tiempo después presenta la Web 2.0, la que incluyó las redes sociales que han sido de gran impacto en todos los sectores incluyendo el sector comercial y económico (Hütt-Herrera, 2012).

El Internet y la web 2.0 abrió una gran variedad de formas de comunicación la mismas que en poco tiempo extendió por todo el mundo una nueva forma de comunicarse por medio de las redes sociales, las cuales han sido una herramienta eficaz para la difusión del comercio, permitiendo a nuevos emprendedores a ofrecer sus productos, bienes y servicios a gran escala, pues la presencia de las empresas en el entorno de las redes sociales les permite dar a conocer al mercado su marca y sus productos obteniendo resultados a corto y mediano plazo (Orihuela \& Colliva, 2008).

Así mismo, las redes sociales pueden ser implementadas con la intención de generar identidad corporativa de la empresa (Del-Prete \& Redon-Pantoja, 2020), siendo necesario promover la filosofía organizacional en función de proyectar las metas de la organización a través de una visión fundamentada en la consecución de elevar la rentabilidad siendo considerable tener en cuenta que el internet de las cosas permite diseñar una economía 
Revista Arbitrada Interdisciplinaria KOINONIA

Año VI. Vol VI. N 12. Julio - Diciembre. 2021

Hecho el depósito de Ley: FA2016000010

ISSN: $2542-3088$

FUNDACIÓN KOINONIA (F.K). Santa Ana de Coro. Venezuela.

María Laura Arteaga-García; María Hercilia Menéndez-Mera

digital en consideración de insertarse a una comunidad global que cada vez se consolida como mercado trasnacional (Bricio-Samaniego et al. 2018).

En razón de lo planteado, la investigación tiene por objetivo analizar las redes sociales como recurso innovador para emprendedores de la parroquia Andrés de Vera del cantón Portoviejo, provincia de Manabí-Ecuador.

\section{METODOLOGÍA DE LA INVESTIGACIÓN}

La metodología empleada es de tipo descriptiva con diseño no experimental, con la intención de conocer tal como ocurren los hechos, sin manipulación deliberadamente, de ese modo, se describieron las características evidenciadas a través de los datos obtenidos.

La población fue de tipo finita conformada por 140 emprendedores de la parroquia Andrés de Vera del cantón Portoviejo, provincia de Manabí-Ecuador, siendo accesible a los investigadores, se tomó en su totalidad como muestra poblacional con la finalidad de contar con mayor validez de los datos recopilados.

La técnica empleada para la recopilación de los datos fue la encuesta online, mientras como instrumento se aplicó un cuestionario en escala Likert con cinco alternativas de respuestas, el cual fue validado por el juicio de tres expertos y cálculo de confiabilidad Alfa de Cronbach con un valor de 0.89 siendo considerable confiable de su aplicación. Una vez obtenido los datos, se organizaron en el programa Excel con la intención de calcular la frecuencia, porcentaje, de los indicadores investigados, de ese modo, se procesaron mediante estadística descriptiva, siendo presentados en tablas en la sección de resultados.

\section{RESULTADOS}

En razón de los datos analizados, se presentan los resultados de la investigación, con la intención de describir la frecuencia de uso de las redes sociales por parte de la muestra poblacional: 
Revista Arbitrada Interdisciplinaria KOINONIA

Año VI. Vol VI. N 12 . Julio - Diciembre. 2021

Hecho el depósito de Ley: FA2016000010

ISSN: 2542-3088

FUNDACIÓN KOINONIA (F.K). Santa Ana de Coro. Venezuela.

María Laura Arteaga-García; María Hercilia Menéndez-Mera

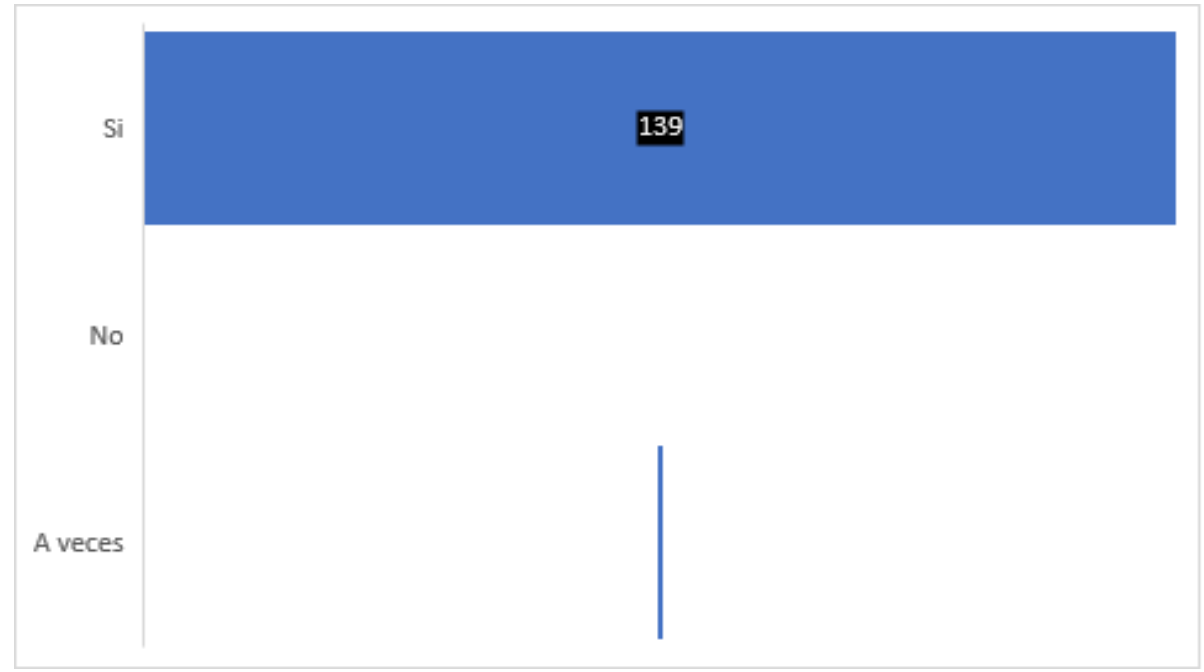

Gráfico 1. Utilidad de las redes sociales en confinamiento por COVID-19.

Fuente: Encuestas.

En el grafico 1, se evidencia que 139 emplearon las redes sociales para ofertar sus productos o servicios, durante el periodo por confinamiento de la pandemia del Covid-19.

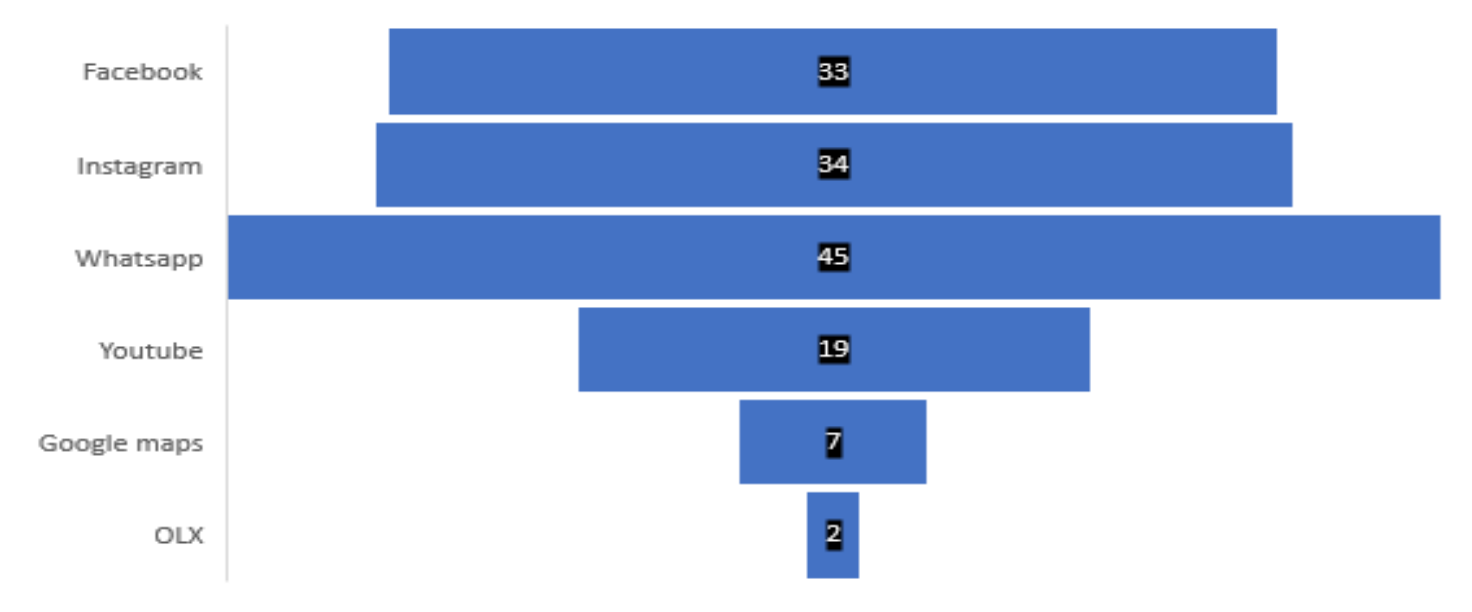

Gráfico 2. Redes sociales más utilizadas por los emprendedores. Fuente: Encuestas. 
El gráfico 2, indica que las redes sociales como innovación virtual en la economía es liderada por Facebook, Instagram, WhatsApp, como medios más rápido y eficaz para ofertar los productos y servicio de los emprendedores de la Parroquia Andrés de Vera de la ciudad de Portoviejo.

\section{DISCUSIÓN}

Los resultados concuerdan con la visión de (Del-Prete \& Redon-Pantoja, 2020) y (Herrera-Echeverri, 2009), sobre la importancia de gestionar el emprendimiento por las redes sociales, por cuanto son una vía que conlleva a la visibilización de la empresa en razón de ser conocida, además de ser en medio de la pandemia por COVID-19, una oportunidad para lograr un mercado que se encuentra incierto por cuanto se articula en base a las necesidades económicas de la población, priorizando el orden de compras en razón de lo que considere urgente, necesario, en razón de prever ahorros o respaldo para cuidados de la salud por lo sensible que se encuentra a la luz de la pandemia (LizarasoCaparó \& Del-Carmen-Sara, 2020).

En este sentido, (González-Torres et al. 2020), confirman que la economía se ha visto por la pandemia, ante lo cual, los emprendedores deben responder a las diversas inquietudes del consumidor, siendo un factor a tener en cuenta, la credibilidad de los servicios, productos, ofertados, por cuanto el principal temor radica en no ser estafados, sobre todo cuando se trata de comercios pequeños o no conocidos, visión compartida en la figura 1. 
Revista Arbitrada Interdisciplinaria KOINONIA

Año VI. Vol VI. N 12 . Julio - Diciembre. 2021

Hecho el depósito de Ley: FA2016000010

ISSN: 2542-3088

FUNDACIÓN KOINONIA (F.K). Santa Ana de Coro. Venezuela.

María Laura Arteaga-García; María Hercilia Menéndez-Mera

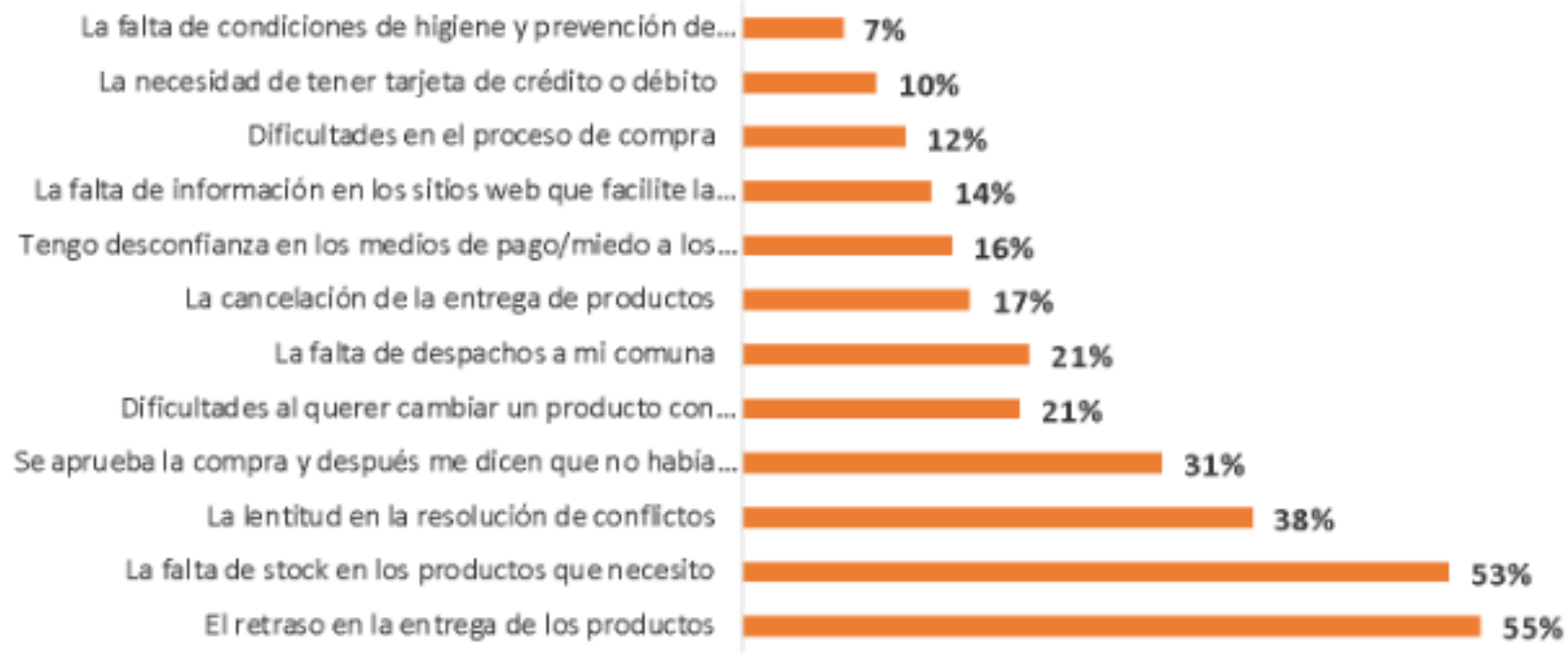

Figura 1. Temores de consumidor al elegir comprar por internet. Fuente: Del-Villar (2020).

La figura 1, ilustra una serie de dificultades que deben prever los emprendedores para promover un negocio efectivo a través de las redes sociales, siendo considerable que no solo se trata de vender un determinado producto, sino, que a la luz de la pandemia se hace necesario contabilizar una serie de elementos que contribuyen a promover confianza y fidelidad en el cliente con la intención de permanecer, crecer, en un mercado competitivo como el actual, siendo considerable para tal fin, tener en cuenta la investigación de (Sánchez-Alzate \& Montoya-Restrepo, 2016), donde se corrobora que uno de los principales elementos a tener en cuenta es la seguridad informática y de datos del consumidor a la hora de realizar las compras online. Así mismo, en la figura 2, se muestran los insumos de mayor demanda. 
Revista Arbitrada Interdisciplinaria KOINONIA

Año VI. Vol VI. N 12. Julio - Diciembre. 2021

Hecho el depósito de Ley: FA2016000010

ISSN: 2542-3088

FUNDACIÓN KOINONIA (F.K). Santa Ana de Coro. Venezuela.

María Laura Arteaga-García; María Hercilia Menéndez-Mera

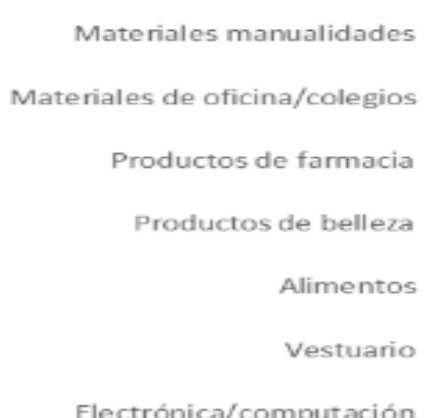

$$
\begin{array}{|l|}
\hline 12 \% \\
\hline 20 \% \\
\hline 25 \% \\
\hline 28 \% \\
\hline 54 \% \\
\hline 54 \% \\
\hline 55 \% \\
\hline
\end{array}
$$

Figura 2. Rubros más compraos por internet.

Fuente: Del-Villar (2020).

La figura 2, evidencia los rubros de mayor venta durante el primer periodo por confinamiento de la pandemia por COVID-19, lo cual, permite tener una visión de mercado para que los emprendedores tomen acciones en razón de trabajar en ser productivos a la hora de ofertar sus emprendimientos, siendo considerable potenciar elementos como el marketing digital a través de las redes sociales para potenciar las posibles compras por parte de los usuarios (Miranda-Zavala \& Cruz-Estrada, 2016). Por otro lado, en la figura 3 , se muestran los elementos a tener en cuenta para mayor seguridad de ventas a través de las redes sociales.

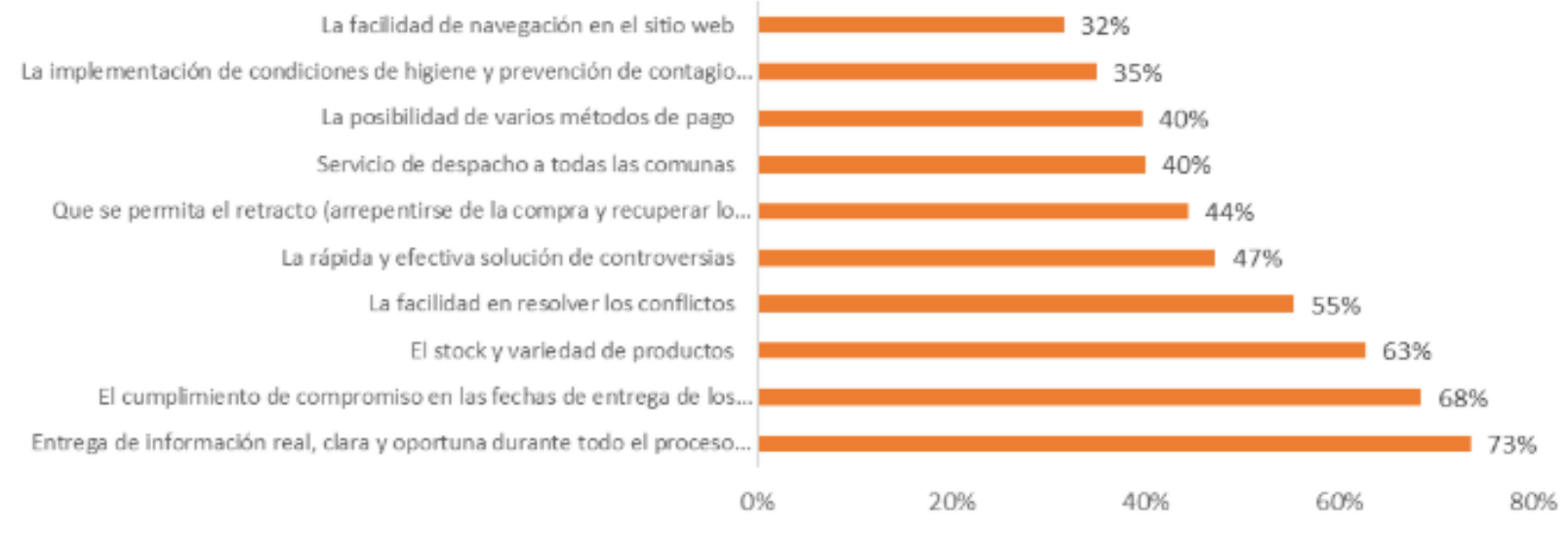

Figura 2. Factores a resguardar en compras por internet.

Fuente: Del-Villar (2020). 
La figura 2 destaca factores a tener en cuenta para elevar la confianza y credibilidad del usuario a la hora de experimentar una compra online, entre las cuales (Sánchez-Alzate \& Montoya-Restrepo, 2016), se encuentran: "la reputación del vendedor, el riesgo percibido, la privacidad de los datos, la seguridad en la transacción, la asociación, y el género del comprador" (p. 160). Superar los problemas descritos en las figuras anteriores, es necesario para que los emprendedores online a través de las redes sociales, puedan gestionar un rendimiento efectivo de sus ideas de negocio.

En este sentido, (Atarama-Rojas \& Vega-Foelsche, 2020), recomiendan el Facebook como página para gestionar una imagen corporativa en las redes sociales, de ese modo, se gestiona mayor acercamiento con el público, por cuanto es una plataforma si se quiere de las tradicionales, por lo tanto, se hace manejable para usuarios de edad, los cuales, no se encuentran familiarizados con lo tecnológico, siendo este un elemento a tener en cuenta por los emprendedores, por cuanto cada segmento poblacional se ve identificado con una red social en particular, siendo necesaria a diversificación, pero manteniendo identidad corporativa para generar credibilidad y confianza en el comprador.

Así mismo, Instagram, se constituye en una plataforma que permite no solo promover el negocio, sino, interactuar socialmente con los clientes, por lo tanto, se abre una oportunidad para pasar de un mero comercio electrónico a uno social (Guzmán-Duque \& Abreo-Villamizar, 2017), por lo que es necesario que los emprendedores se organicen en un gremio que les permita promoverse a través de las redes sociales, gestionando mayor credibilidad en el usuario, siendo significativo cuando existe un volumen importante de personas navegando por las redes sociales (Alderete \& Jones, 2019).

En complemento, (Guzmán-Duque, 2018), refiere que s-commerce, sea promovida por los emprendedores, pero para ser efectivos, deben idear sus estrategias de venta en conformidad a las redes sociales en particular, aunado a la necesidad de tener en cuenta la contratación laboral y el modo de cumplir los acuerdos patronales a fin de crecer en el tiempo desde una idea de emprendimiento. 
Otro aspecto a tener en cuenta por los emprendedores, es que se encuentran frente a la generación millennials, siendo esta habituada al uso de las redes sociales, siendo que deben combinar entre el estilo clásico de los usuarios y este segmento poblacional, de ese modo, se contextualiza en razón de promover un mercado integral que permita adaptarse al tiempo, teniendo en consideración que las nacientes generaciones se basaran en el internet de las cosas (Anaya-Sánchez et al. 2020), siendo además que una efectiva estrategia de venta, contribuye en la toma de decisión de la población millennials.

\section{CONCLUSIÓN}

El principal reto de los emprendedores, es posicionar su negocio en una sociedad competitiva, donde las marcas consolidadas, tienen la primera opción de compra, por lo tanto, deben diseñar estrategias de venta, pero sobre todo de confianza, credibilidad, para atraer al público, siendo necesario exponer la seguridad de los datos del cliente, atención de calidad, posibilidad de cambiar el producto si no se está conforme, devolución de dinero de forma rápida, entrega a domicilio, son algunos de los elementos a considerar, además que Facebook, Instagram, figuran como las plataformas de mayor uso por parte de los usuarios o potenciales compradores.

\section{FINANCIAMIENTO}

No monetario

\section{AGRADECIMIENTOS}

Al Instituto Tecnológico Superior Paulo Emilio Macías por el impulso en el desarrollo de la investigación. 
Revista Arbitrada Interdisciplinaria KOINONIA

Año VI. Vol VI. N 12. Julio - Diciembre. 2021

Hecho el depósito de Ley: FA2016000010

ISSN: 2542-3088

FUNDACIÓN KOINONIA (F.K). Santa Ana de Coro. Venezuela.

María Laura Arteaga-García; María Hercilia Menéndez-Mera

\section{REFERENCIAS CONSULTADAS}

Alderete, M, \& Jones, C. (2019). ¿Hacia el social commerce? El valor de las redes sociales en la MiPyME de Córdoba, Argentina [Towards social commerce: the value of social networks for Micro and SME from Córdoba, Argentina]. Entramado, 15(1), 48-60. https://doi.org/10.18041/1900-3803/entramado.1.5149

Anaya-Sánchez, R., Castro-Bonaño, J. M., \& González-Badía, E. (2020). Preferencias del consumidor millennial respecto al diseño de webs de social commerce [Millennial consumer preferences regarding social commerce website design]. RBGN - Revista Brasileira De Gestão De Negócios, 22(1), 123-139. https://doi.org/10.7819/rbgn.v22i1.4038

Atarama-Rojas, T, \& Vega-Foelsche, D. (2020). Comunicación corporativa y branded content en Facebook: un estudio de las cuentas oficiales de las universidades peruanas [Corporate communications and branded content on Facebook: A study of the official accounts of Peruvian universities]. Revista de Comunicación, 19(1), 3753. https://dx.doi.org/10.26441/rc19.1-2020-a3

Bricio-Samaniego, K, Calle Mejía, J, \& Zambrano Paladines, M. (2018). El marketing digital como herramienta en el desempeño laboral en el entorno ecuatoriano: estudio de caso de los egresados de la Universidad de Guayaquil [Digital marketing as a tool for labor performance in the Ecuadorian Environment: Case study of graduates from the University of Guayaquil]. Revista Universidad y Sociedad, 10(4), 103-109.

Del-Prete, A, \& Redon-Pantoja, S. (2020). Las redes sociales on-line: Espacios de socialización y definición de identidad [Virtual social networks: Spaces of socialization and definition of identity]. Psicoperspectivas, 19(1), 86-96. https://dx.doi.org/10.5027/psicoperspectivas-vol19-issue1-fulltext-1834

Del-Villar, L. (2020). Casi un 90\% de las personas que han comprado por Internet durante la pandemia admiten haber tenido problemas [Almost $90 \%$ of people who have bought online during the pandemic admit to having had problems]. Recuperado desde https://n9.cl/94e7l 
Garzón-Sánchez, I. (2017). Diseño de un plan de marketing en redes sociales (Facebook, Instagram, Twitter, Whatsapp, Youtube) para incrementar las ventas en el centro comercial "parqueadero la merced" ubicado en el sector centro del distrito metropolitano de Quito 2016-2017 [Design of a marketing plan in social networks (Facebook, Instagram, Twitter, Whatsapp, Youtube) to increase sales in the shopping center "parqueadero la merced" located in the central sector of the metropolitan district of Quito 2016-2017]. https://dspace.cordillera.edu.ec/handle/123456789/2989

González-Torres, I, Collado Cardoso, E, Torres Mora, I, Quiroga Gómez, Z, Giraldo, C, \& Gómez Rosabal, A. (2020). Efecto económico parcial de la COVID-19 y sus resultados en Camagüey, Cuba [Partial Economic Effect of COVID-19 in Camagüey, Cuba]. Retos de la Dirección, 14(2), 33-53.

Guzmán-Duque, A. (2018). El S-commerce: La innovación a través de Medios Sociales [S-commerce: Innovation through Social Media]. Journal of technology management \& innovation, 13(1), 56-65. https://dx.doi.org/10.4067/S0718-27242018000100056

Guzmán-Duque, A. P., \& Abreo-Villamizar, C. A. (2017). Del comercio electrónico al comercio social: La innovación al alcance de las organizaciones. Estudio para el sector calzado Bucaramanga, Colombia [From electronic commerce to social commerce: Innovation within the reach of organizations. Study for the footwear sector Bucaramanga, Colombia]. Contabilidad Y Negocios, 12(24), 107-118.

Herrera-Echeverri, H. (2009). Investigación sobre redes sociales y emprendimiento: revisión de la literatura y agenda futura [Research into social and entrepreneurship networks: a literature review and future agenda]. Innovar, 19(33), 19-33.

Hütt-Herrera, H. (2012). Las redes sociales: una nueva herramienta de difusión [Social networks: a new dissemination tool]. Reflexiones, 91(2),121-128.

Lizaraso-Caparó, F, \& Del-Carmen-Sara, J. (2020). Coronavirus y las amenazas a la salud mundial [Coronaviruses and global health threats]. Horizonte Médico (Lima), 20(1), 4-5. https://dx.doi.org/10.24265/horizmed.2020.v20n1.01

Miranda-Zavala, A., \& Cruz-Estrada, I. (2016). Redes sociales: Herramienta de Marketing Internacional en el sector hotelero [Social networks: International Marketing Tool in the hotel sector]. El Periplo Sustentable, (31).

Orihuela-Colliva, J. L. (2008). Internet: la hora de las redes sociales [Internet: time for social media]. Recuperado desde https://n9.cl/sfizl 
Revista Arbitrada Interdisciplinaria KOINONIA

Año VI. Vol VI. N 12. Julio - Diciembre. 2021

Hecho el depósito de Ley: FA2016000010

ISSN: $2542-3088$

FUNDACIÓN KOINONIA (F.K). Santa Ana de Coro. Venezuela.

María Laura Arteaga-García; María Hercilia Menéndez-Mera

Sánchez-Alzate, J, \& Montoya-Restrepo, L. (2016). Factores que afectan la confianza de los consumidores por las compras a través de medios electrónicos [Factors that affect consumer confidence for purchases through electronic means]. Pensamiento \& Gestión, (40), 159-183. https://doi.org/10.14482/pege.40.8809

(O2021 por los autores. Este artículo es de acceso abierto y distribuido según los términos y condiciones de la licencia Creative Commons Atribución-NoComercial-Compartirlgual 4.0 Internacional (CC BY-NC-SA 4.0)

(https://creativecommons.org/licenses/by-nc-sa/4. 